\title{
LAS DEMASIADAS APLICACIONES: PARÁMETROS E INDICADORES PARA SELECCIONAR LAS TOPAPP DE LECTURA PARA NIÑOS
}

\author{
Araceli García-Rodríguez* \\ Facultad de Traducción y Documentación. Universidad de Salamanca. \\ Raquel Gómez-Díaz** \\ Facultad de Traducción y Documentación. Universidad de Salamanca.
}

\begin{abstract}
Resumen: Partiendo de la necesidad de sistematizar criterios que permitan la evaluación de las aplicaciones de lectura para niños de una forma objetiva, el artículo propone un listado de indicadores estructurados en parámetros que a su vez se agrupan en las dimensiones de forma y contenido. La dimensión forma incluye la disponibilidad, adquisición, seguridad, privacidad, popularidad, reconocimiento, usabilidad y ergonomía. La de contendido abarca los indicadores relacionados con la autoría, actualización, accesibilidad, organización, personalización e interacción con los contenidos así como la calidad del audio y la interactividad. Como resultado y aplicación práctica el artículo propone una plantilla que recoge los aspectos fundamentales a evaluar y la indicación de aquellos que son recomendables u obligatorios de cara a facilitar la selección de aplicaciones de lectura infantil.

Palabras clave: Aplicaciones de lectura infantil; evaluación de aplicaciones de lectura; lectura digital infantil; indicadores de evaluación de app.

Title: TOO MANY APPS: BENCHMARKS AND INDICATORS TO SELECT TOPAPP CHILDREN READING APP.

Abstract: Based on the need to systematize criteria for evaluating applications reading for children, in an objective manner the article proposes a list of indicators structured parameters which in turn are grouped into the dimensions of form and content. The form dimension includes the availability, acquisition, security, privacy, popularity, recognition, usability and ergonomics. Contended the covers of indicators related to authorship, update, accessibility, organization, customization and interaction with content and audio quality and interactivity. As a result, practical application the article proposes a template that includes the fundamental aspects and evaluate the indication of those that are recommended or required in order to facilitate the selection of children's reading applications.

Keyword: Kids Reading apps; reading apps evaluation; kids digital reading; evaluation indicators of app.
\end{abstract}

\section{INTRODUCCIÓN: LA EVALUACIÓN DE LAS APLICACIONES DE LECTURA DIGITAL}

Zaid (2010) decía que cada 30 segundos se publicaba un libro. No sabemos si cada 30 minutos o tal vez una vez día, sale al mercado una app para niños. ¿ंHay demasiadas?, ¿cómo distinguir las buenas de las mediocres y las malas? Precisamente la intención de este artículo es establecer indicadores que ayuden a la evaluación de apps de lectura infantil para después poder seleccionar.

Se entiende por aplicaciones de lectura aquellos "programas informáticos que permiten la gestión de contenidos (...) y el acceso a los mismos, así como la interacción con el lector usuario, facilitando en la mayor parte de los casos la adaptación del contenido (...) para ser leído en un dispositivo móvil de lectura” (Gómez-Díaz y Cordón-García, 2015) y que integran los contenidos de varios libros en el mismo producto.

Las aplicaciones para niños han experimentado un desarrollo espectacular en los últimos años y aunque en menor medida, las aplicaciones de lectura dedicadas a ellos también comienzan a ser numerosas. Cada vez hay más hogares que disponen de tabletas ${ }^{1}$ y los niños comienzan a utilizarlas cada vez a edades más tempranas, por lo que el mercado de aplicaciones para los más pequeños en general y, en particular, las de lectura están experimentando un crecimiento que se puede observar en el aumento de la oferta y en el incremento de la venta de dispositivos. Por eso, no puede extrañarnos que los consumidores de este tipo de literatura (niños, padres, maestros y bibliotecarios) y los propios editores y desarrolladores, demanden cada vez más puntos de referencia que les permitan adquirir y publicar productos interesantes, encontrándose entonces con la falta de criterios específicos para la edición digital.

\footnotetext{
*araceli@usal.es

** rgomez@usal.es
}

Recibido: 14-05-2015; 2a versión: 16-06-2015; aceptado: 26-09-2015.

GARCÍA RODRÍGUEZ, A. y GÓMEZ DÍAZ, R. Las demasiadas aplicaciones: parámetros e indicadores para seleccionar las topapp de lectura para niños. Anales de Documentación, 2015, vol. 18, $\mathrm{n}^{\circ}$ 2. Disponible en: http://dx.doi.org/10.6018/analesdoc.18.2.227071. 
La selección de literatura infantil se realiza desde hace años aplicando una serie de criterios, parámetros e indicadores aceptados por la mayoría de los especialistas, para ello, pueden consultarse los trabajos de Lluch (2009 y 2010) y Colomer (2010). Al igual que con los libros en papel los gustos e intereses del lector han de estar muy presentes en nuestra labor de selección, porque si los obviamos difícilmente podremos conseguir nuestros objetivos: motivar en la lectura, desarrollar el gusto y el hábito lector y sobre todo que los más pequeños disfruten con la lectura.

Además, en el mundo digital son necesarios otros criterios que afectan no solo al contenido textual, sino también al audio, la animación, el diseño, al formato, la interactividad, a su visibilidad, valoración, usabilidad, etc. Nos encontramos pues, ante objetos que es preciso analizar de forma diferenciada, articulando parámetros e indicadores propios que nos permitan determinar sus características, requisitos y calidad. Evaluar nos permitirá establecer el valor de una aplicación de lectura para niños, proceso para el cual es necesario conocer previamente sus características y funcionalidades.

Como ya hemos mencionado, la cantidad y variedad de aplicaciones es grande.

En julio de 2015, según el informe realizado por Statista² ${ }^{2}$, solo entre Google Play y Apple Store, había en el mercado más de tres millones de aplicaciones, siendo las más populares los juegos, seguidos de las educativas y las de entretenimiento, ocupando los libros el octavo lugar. ${ }^{3}$ El elevado número de aplicaciones, entre ellas las de lectura y las múltiples posibilidades de estas, hacen necesario tener en cuenta multitud de variables y contar con una herramienta que facilite, en base a unos determinados parámetros, su comparación. Para determinar la calidad de una app no es suficiente con descargarla y probarla, es necesaria una metodología adecuada basada en la definición de criterios, parámetros e indicadores. Los parámetros nos permitirán determinar qué es lo que queremos evaluar, mientras que los indicadores nos servirán para determinar cómo vamos a hacerlo y medir cada uno de ellos, sin olvidarnos que cada parámetro puede generar varios indicadores y que algunos indicadores pueden ayudarnos a medir más de un parámetro.

Este trabajo es sólo el primero de una serie en la que pretendemos proponer indicadores para otro tipo de productos digitales como son los libros app y los audiolibros.

\section{OBJETIVOS}

Entre tantas aplicaciones de lectura disponibles en el mercado nos encontramos con productos de desigual calidad, por lo que es necesario conocer, evaluar y seleccionar críticamente los recursos y articular sistemas de recogida de información y valoración que nos permitan elegir las mejores. Por ese motivo, los objetivos fundamentales de este artículo son:

- Destacar la importancia de la tarea de selección de contenidos digitales para niños

- Establecer criterios y parámetros de selección en torno a los que debe articular el análisis y la evaluación de aplicaciones de lectura

- Diseñar herramientas de trabajo para su selección de cara a la puesta en marcha de un servicio de recomendación de aplicaciones en las bibliotecas infantiles, al igual que se viene haciendo tradicionalmente con los libros impresos

- Facilitar la tarea de selección y valoración a padres, docentes y bibliotecarios

\section{METODOLOGÍA PARA LA EVALUACIÓN DE APLICACIONES DE LECTURA}

La recomendación de títulos bien directamente o a través de guías de lectura, ha sido, y es, una actividad propia de maestros y bibliotecarios. En un momento en el que la lectura digital entre niños y jóvenes parece ir avanzando poco a poco, su tarea como recomendadores puede ir más allá con la creación de propuestas innovadoras como la elaboración de guías de aplicaciones, tal y como esta haciendo el grupo de investigación Gretel ${ }^{4}$ para libros-app, el Canal Lector ${ }^{5}$ dentro de la sección selecciones temáticas o las guías de de la Fundación Germán Sánchez Ruipérez, para lo cual es necesario asignar criterios, parámetros e indicadores.

La metodología seguida para determinar qué elementos se deben considerar en la evaluación de aplicaciones ha sido establecida en cuatro pasos que se explican a continuación. 


\subsection{Documentación del tema}

Existen diversos trabajos dedicados a la evaluación productos documentales como es el caso de las bases de datos (Rodríguez Yunta, 1998; Rodríguez Yunta y Giménez Toledo, 2004; Guillén Ríos, 2014), los recursos digitales (Codina, 2000), los cibermedios (Rodríguez Martínez, Codina y Pedraza Jiménez, 2012), las interfaces (Rodríguez Bravo y Alvite Díez 2004), o las aplicaciones multimedia (Sánchez et al., 2002), por citar algunos. Pero tras una búsqueda en LISA (Library and Information Science Abstrac), LISTA (Library, Information Science \& Technology Abstracts), SCOPUS, WOS, ERIC y Google Académico utilizando los descriptores “reading app”, “aplicaciones de lectura”, “evaluación aplicaciones de lectura”, “evaluation reading app” hemos constatado que a pesar del crecimiento de las aplicaciones de lectura son pocos los trabajos que se encargan de su evaluación.

Si bien en las citadas búsquedas se han podido localizar algunos estudios que analizan determinadas aplicaciones de lectura como es el de Ho (Ho; Lu; y Lin, 2013) que trata sobre libros y revistas, el de Rello y Baeza-Yates (2014) que aborda el tema de una aplicación para la dislexia, la aproximación a la evaluación (Gómez-Díaz, 2012), los desarrollos de aplicaciones (Sanz y García, 2014), las aplicaciones educativas de Guillem y Celaya (2014), las monografías científicas electrónicas (Mangas-Vega, 2014), la selección de contenidos infantiles (García-Rodríguez, Gómez-Díaz y Cordón-García, 2013) o las recomendaciones sobre las funcionalidades de las aplicaciones de lectura atendiendo a la edad de los lectores (García-Rodríguez, 2014), en la mayoría de los casos se abordan los criterios de evaluación de manera tangencial y genérica o por el contrario demasiado específica, por ejemplo centrándose en las ilustraciones, la interactividad, etc.

Podemos mencionar en este caso los trabajos de Cahil y McGill Frazen (2013) sobre la selección de libros apps de imágenes, el de Bircher, (2012) en el que se recogen algunas recomendaciones y ejemplos de lo que debe ser un buen libro de imágenes en formato app o el de Henning (2014) centrado en los elementos que debe incluir una recomendación incluyendo un cheklist para su selección.

Por todo ello, consideramos que trabajar en este tema es una laguna que hay que tratar de solventar.

\subsection{Búsqueda de elementos de las aplicaciones de lectura infantil y sistematización de la información}

El análisis de múltiples aplicaciones proporcionó una nómina de aspectos relevantes en los que incidir de cara a la evaluación, y los estudios, recursos y fuentes mencionados anteriormente se utilizaron como base metodológica para la definición de parámetros e indicadores. Asimismo se adaptaron algunos indicadores utilizados para la evaluación de otros productos documentales, como bases de datos y páginas web, a las aplicaciones de lectura.

Con el objetivo de poder establecer una sistematización de la información y siguiendo la metodología utilizada por Codina $(2000,2006)$ para la evaluación de recursos digitales en línea, se estructuró la información de forma que combinara los aspectos puramente formales con las cuestiones de contenido, estableciéndose dos dimensiones: forma y contenido. Para cada dimensión se especificaron los parámetros y sus características; los indicadores con su descripción; en los casos necesarios cómo medir dicho indicador e incluso la fuente.

Para facilitar su consulta y aplicación se codificaron las dimensiones, parámetros e indicadores. En lo que se refiere a la puntuación para cada indicador se fueron haciendo las anotaciones pertinentes que pueden consultarse en la plantilla de evaluación.

\subsection{Selección y definición de las dimensiones, parámetros e indicadores}

\subsubsection{La dimensión forma (F)}

Cuando hablamos de lectura interactiva no basta con tener un buen texto y una buena historia sino que también hace falta que esa idea se integre de manera eficiente en el soporte en el que va a ser leída, por lo que, al igual que lo hacemos en la literatura impresa, tendremos que valorar todos los aspectos relativos a las cuestiones formales.

La dimensión forma abarca ocho parámetros: disponibilidad, adquisición, seguridad, confidencialidad y privacidad, popularidad, reconocimiento y ergonomía.

\section{F 1 Disponibilidad}

Utilizamos el término disponibilidad para determinar la facilidad de acceso a la propia aplicación. Sus parámetros son: 


\section{F.1.1 Disponibilidad para diferentes sistemas operativos}

El sistema operativo condiciona la programación y por tanto el desarrollo de la misma. Algunas estan disponibles para los diferentes sistemas y otras son exclusivas de uno determinado. Los más frecuentes son Android e iOS y en menor medida Windows.

A la hora de valorar una aplicación hay que tener en cuenta que si hay muchas diferencias entre el desarrollo en los distintos sistemas operativos es aconsejable evaluarlas de manera independiente.

\section{F.1.2 Disponibilidad en diferentes sitios}

Cada vez son más los desarrolladores que ofrecen sus productos en diferentes recursos y plataformas que enlazan con las tiendas, conscientes, de que esto favorece la disponibilidad y la descubribilidad ${ }^{6}$ de sus aplicaciones.

Se valorará positivamente que el desarrollador cuente con una página web y que la aplicación tenga un enlace directo a la tienda correspondiente. Esto mejora la disponibilidad del producto, facilita el acceso y la descarga.

\section{F.1.3 Disponibilidad de la app en varios idiomas}

Se valorará positivamente que el usuario pueda personalizar la lengua en la que quiere utilizar la aplicación pues de este modo se amplía el número de lectores potenciales. Este indicador solo hace referencia al idioma de uso de la aplicación, independientemente de que también se puedan o no consultar los contenidos en distintos idiomas.

\section{F.1.4 Título identificable}

Los títulos y los iconos de las apps deben ser fácilmente reconocibles, identificables y preferiblemente cortos. La utilización de títulos tan genéricos como "libros infantiles" o "cuentos infantiles", habituales para este público, dificulta no solo la distinción frente a otras similares, sino también la memorización del nombre por parte de los usuarios. $^{7}$

\section{F.1.5 Icono identificable}

El icono es a una aplicación lo que la cubierta a un libro, es su tarjeta de presentación y una forma de distinguirla entre otros muchos productos similares.

Es habitual que el usuario no recuerde el nombre exacto de una aplicación, pero sí que reconozca fácilmente el icono. Este debe ser atractivo, llamar la atención del usuario, estar relacionado con el contenido del producto que identifica o con el personaje y, en el caso de los niños, preferiblemente de colores llamativos y con pocos elementos para que su reconocimiento sea más rápido. Para los bundles, ${ }^{8}$ se recomienda que incluyan una imagen que identifique que se trata de un paquete de aplicaciones para distinguirlo de aquellas que se pueden adquirir de forma individual.

\begin{tabular}{|l|l|l|l|}
\hline Icono & \multicolumn{1}{|c|}{ Título } & Análisis \\
\hline
\end{tabular}




\begin{tabular}{|l|l|l|l|}
\hline & Geronimo Stilton & $\begin{array}{l}\text { Adaptada al personaje } \\
\text { Diseño alegre y colorista } \\
\text { Inconfundible }\end{array}$ \\
\hline
\end{tabular}

Tabla I. Ejemplos de iconos y títulos de apps infantiles.

\section{F.2 Adquisición}

En cuanto a la adquisición, el precio no debería ser un factor definitivo a la hora de descargar una app, aunque esta claro que es el que más se utiliza. No siempre los productos gratuitos cumplen los mínimos criterios de calidad, lo que no quiere decir que no haya aplicaciones baratas e incluso gratis interesantes y adecuadas.

La mayor parte de las aplicaciones de lectura suelen ser gratuitas lo que tendremos que analizar es la forma de adquisición de los contenidos, sin olvidar que el precio puede cambiar en función del sistema operativo y que éstos cambian permanentemente.

F.2.1 Modalidad de adquisición de los contenidos

Existen varias modalidades de adquisición por lo que es necesario determinar cuál es la más adecuada. Son especialmente valorables aquellas que permiten diferentes opciones dejando al lector la elección del sistema que mejor se adapte a sus intereses y necesidades. Las modalidades de adquisición de contenidos son:

- Compras integradas o título a título en este caso se suele incluir un título gratuito y el resto hay que comprarlo. ${ }^{9}$

- Suscripción.

- Gratuita con publicidad: son especialmente rechazables aquellas aplicaciones con publicidad invasiva ya que no solo dificultan, distraen y entorpecen la lectura, sino que en ocasiones la falta de filtros ${ }^{10}$ puede hacer que nos encontremos banners que anuncian casinos, casas de apuestas, servicios de contactos, etc.

- Gratuita sin publicidad: tanto la aplicación como todos sus contenidos versión lite: son las que permiten un acceso limitado o bien al contenido o a las funcionalidades de la aplicación. Son muy útiles para comprobar el contenido, funcionamiento y sobre todo para decidir su posterior compra.

- Ofertas: incluyen descuentos por compra conjunta o pack y los que se realizan en fechas determinadas: Día del libro, Black friday, aniversario de la empresa...

\section{F.3 Seguridad}

La seguridad más que un parámetro debería ser un requisito indispensable de cualquier aplicación dirigida a menores, por lo que este apartado será evaluado de la forma más detallada posible. Los indicadores propuestos son:

\section{F.3.1 Bloqueo de compras en la propia aplicación}

Aunque existe la opción de bloquear las compras desde el propio dispositivo, en aquellas que siguen el modelo de compras integradas, se valorará positivamente la existencia del bloqueo de compras específico en la app.

\section{F.3.2 Control parental en la propia aplicación}

Teniendo en cuenta que algunas aplicaciones permiten el acceso a internet es recomendable la existencia de un sistema de control parental propio de la aplicación, aparte del que pueda tener el dispositivo para evitar tener que cambiar la configuración de todo el sistema. Resulta de gran utilidad cuando la tableta es compartida por niños y adultos.

\section{F.3.3 Adaptación de las claves de acceso a la edad}

No todas las aplicaciones utilizan los mismos sistemas de control. Es posible que se solicite una clave, la resolución de una pregunta o realizar una acción en la pantalla. Comprobaremos en este caso cuál es el mecanismo y si es el adecuado para la edad de los potenciales lectores. Por ejemplo no es adecuado que una aplicación dirigida a niños de 8 a 9 años proponga como clave de acceso una operación como 26 + 25, pero esta misma suma puede ser válida en otra pensada para niños de 4 o 5 años. 


\section{F.4 Confidencialidad y privacidad}

Todas las aplicaciones, y mucho más las infantiles, deben cumplir las leyes vigentes en cada país de origen sobre la protección de datos, garantizar la confidencialidad y proteger la privacidad de la información ofrecida por los usuarios, datos que deben aparecer de una forma clara y explícita en el producto. ${ }^{11}$

\section{F.4.1 Explicación clara de la política de privacidad}

Es necesario comprobar, a través de la web del desarrollador, la tienda de aplicaciones o el propio producto, si la política de privacidad está claramente especificada y aparece de forma explícita qué datos se solicitan y cuáles se recogen de forma automática (dirección IP, identificación del dispositivo...), el uso de esos datos y la garantía de confidencialidad.

\section{F.4.2 Anonimato de los menores}

Las aplicaciones para niños no deben solicitar ningún tipo de información personal de los menores para activar su funcionamiento y mucho menos sin el consentimiento del adulto.

\section{F.5 Popularidad}

La popularidad es un indicador que nos permite determinar la importancia de una aplicación y su frecuencia de uso, equivaldría al número de seguidores en una red social o el número de visitas de una web. Una aplicación con un elevado número de descargas, con una buena puntuación y una buena valoración, tiene más posibilidades de ser descargada.

Dentro de la popularidad se puede considerar el número de descargas que, aunque no es un indicador directo de calidad, sí puede dar pistas sobre su popularidad. Sin embargo, dada la dificultad para determinar el número de descargas adecuado y debido a que los datos cambian diariamente no se puede utilizar como indicador.

El número de comentarios de las aplicaciones infantiles de lectura suele ser escaso y en la mayoría de los casos hechos por los adultos, pero su consulta y lectura nos da pistas sobre su funcionamiento e interés para los potenciales usuarios. Se recomienda consultar dichos comentarios aunque las valoraciones hay que considerarlas con precaución dada su subjetividad. Los comentarios excesivamente genéricos, no aportan ninguna información.

Este criterio debe ser tomado con precaución porque los comentarios pueden tener distintas motivaciones y las descargas estar impulsadas por las campañas de márketing.

\section{F.5.1 Puntuación de los lectores}

Las puntuaciones recibidas también pueden dar pistas sobre el éxito de una aplicación y la valoración por parte de los lectores, por lo que comprobar en la tienda de aplicaciones cual ha sido la puntuación media obtenida será casi un paso obligatorio.

En este caso habrá que tener en cuenta no solo la calificación media sino también sobre cuantas puntuaciones, pues no es lo mismo tener una puntuación de 5 sobre 3, que sobre 10 valoraciones. Se aconseja la valoración positiva de aquellas apps que hayan recibido una puntuación igual o superior a tres estrellas o puntos sobre un mínimo de 5 valoraciones.

Recomendamos igualmente una cierta flexibilidad pues pueden darse casos, por ejemplo una novedad, que no incluyan este mínimo porque no haya dado tiempo a usarla y valorarla.

\section{F.6 Reconocimiento}

Además del reconocimiento social (popularidad) es conveniente analizar el reconocimiento profesional a través de los premios y sellos de calidad. Esta información se puede obtener en la descripción del propio producto, en la web del desarrollador o de la institución que otorga el premio.

\section{F.6.1 Premios recibidos}

Frente a la gran cantidad de galardones que se otorgan a la literatura impresa, todavía son pocos los premios centrados en este tipo de productos aunque hay algunos que han alcanzado un gran prestigio en poco tiempo como el Mobile for Good Europe Awards, Bologna Digital Awards <http://www.bookfair.bolognafiere.it/bologna-ragazzidigital-award/1034.html>, El Chupete <http://www.elchupete.com/en/home>, Best Mobile App Award $<$ http://www.bestmobileappawards.com/> o el Premio Tab Innovación <http://www.tabinnovation.com/>. 


\section{F.6.2 Sellos de calidad obtenidos}

Un sello de calidad es un logo o símbolo que un organismo o entidad otorga a aquellos productos que cumplen unas determinadas características o requisitos. Los sellos son un aval de su calidad, les aportan un valor añadido y favorecen su visibilidad.

En el caso de las aplicaciones para niños estos sellos suelen hacer referencia a temas tan importantes como la seguridad y adaptación de los contenidos.

\section{F.7 Usabilidad}

La usabilidad es la "capacidad de un software de ser comprendido, aprendido, usado y ser atractivo para el usuario, en condiciones específicas de uso" (ISO/IEC 9126), una definición que hace referencia a los aspectos internos y externos del producto, entendiendo que la usabilidad no depende solo de este sino también del usuario.

Partiendo de este concepto, podemos decir que la usabilidad es el grado en el que los usuarios interactúan con una aplicación de manera eficiente e intuitiva, una característica que está relacionada con la facilidad de uso, con la rapidez con la que podemos aprender a utilizarla y con el grado en el que su diseño facilita o dificulta su manejo.

Al igual que en casos anteriores este parámetro está directamente relacionado con otros como la accesibilidad o la ergonomía (el uso de una tipografía correcta, la utilización de botones fáciles de identificar, etc.), pero además podemos aplicar unos indicadores específicos.

\section{F.7.1 Velocidad}

Hace referencia al tiempo de apertura de la aplicación, un aspecto especialmente valorado.

Las aplicaciones deben abrirse de una forma rápida, entre 10 y 25 segundos es lo recomendable e incluir, durante los tiempos muertos, mensajes sobre el proceso de descarga (loading, descargando,...).

\section{F.7.2 Navegación}

Partiremos en este caso de un principio fundamental, la mejor app es aquella en la que el usuario aprende como se navega en un corto espacio de tiempo. Por ello, la navegación debe ser sencilla e intuitiva en la que el lector tenga siempre el control sobre su funcionamiento.

Un funcionamiento correcto es posible gracias a: botones operativos, acciones sencillas de realizar, y por supuesto, a un producto que no se cuelgue constantemente.

Asimismo pueden colaborar a la usabilidad los iconos de los botones de acción cuando son fácilmente identificables, de un tamaño correcto, y ubicados en lugares visibles.

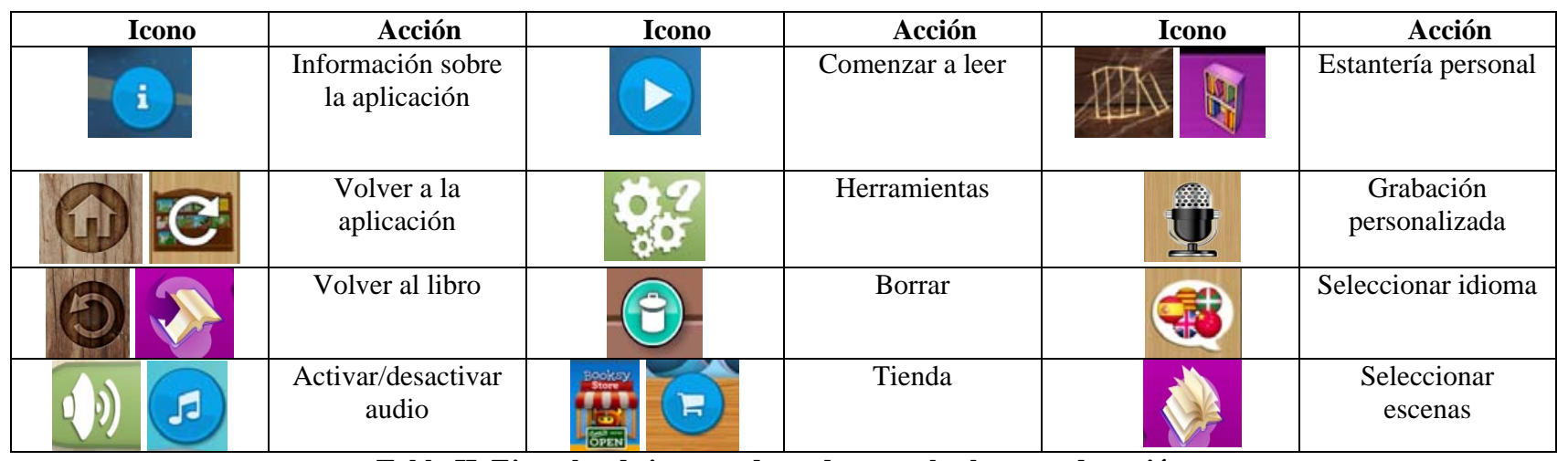

Tabla II. Ejemplos de iconos adecuados para los botones de acción.

Pese a todo lo sencillo que pueda ser el funcionamiento de una app es aconsejable que incluya ayudas o tutoriales con instrucciones claras sobre su uso, el significado de cada botón o icono, siempre utilizando un lenguaje comprensible para los potenciales lectores. En caso de usar términos como "tap", “doble tap”, "drag”, etc., conviene incluir una explicación visual de cada uno de los gestos con su nombre correspondiente. 
La posibilidad de acceso al menú desde todas las páginas es necesaria para que el lector tenga siempre presente la estructura general. Todas las aplicaciones disponen de un menú o barra de herramientas para poder realizar las diferentes acciones. Este tiene que estar en todas las páginas aunque se pueda ocultar o mostrar según el interés del lector.

\section{F.8 Ergonomía}

Se incluyen en este parámetro todos aquellos aspectos que hay que tener en cuenta para visualizar la app de una forma sencilla y clara: el tamaño y el tipo de letra, la colocación de los diferentes elementos e iconos dentro de la página, la claridad de las imágenes, etc.

\section{F.8.1 Legibilidad}

Es la facilidad de lectura del texto de la app (no nos estamos refiriendo al tamaño de la letra de los libros que en muchas ocasiones el propio lector puede personalizar, sino a las pantallas principales de la propia app), algo relacionado básicamente con el tamaño y tipo de letra.

\section{F.8.2 Claridad}

Hace referencia al contraste de la letra con el fondo. Si el fondo es un tono claro, los textos deben ser oscuros y viceversa.

\section{F.8.3 Uso del color}

El uso adecuado de los colores y la utilización de una gama uniforme y reducida, puede colaborar en la legibilidad de los elementos de la pantalla. Por ello son preferibles aquellas que utilizan colores suaves y en las que siempre destaque un color determinado.

\subsubsection{La dimensión contenido (C)}

Los parámetros incluidos en esta dimensión hacen referencia a: autoría, actualización, contenido, accesibilidad, organización, personalización, interacción, calidad del audio e interactividad.

\section{C.1 Autoría}

Determinaremos con este parámetro el prestigio y/o especialidad de la empresa encargada del desarrollo y, si fuera el caso, el de la colección de la que forma parte.

\section{C1.1 Mención del editor/desarrollador}

Con este indicador se trata de establecer con claridad la reputación y responsabilidad de la empresa que desarrolla el producto, dato que debe aparecer claramente reflejado en la tienda y en los títulos de crédito de la propia aplicación.

\section{C.2 Actualización}

Nos permitirá comprobar la novedad de una aplicación y también las modificaciones que se van haciendo en las distintas versiones. Los indicadores a utilizar son:

\section{C.2.1 Especificación de la fecha de creación}

La fecha de creación puede aportar pistas sobre su éxito, su uso y su permanencia en el mercado en el caso de las más antiguas, pero también permitirá identificar novedades cuando son de fecha más reciente.

Tendremos en cuenta en este caso la fecha de creación original o de la primera versión. En App Store esta información se puede consultar en el "historial de versiones", Google Play solo ofrece la fecha de la última actualización, mientras que la tienda de aplicaciones de Amazon es la única que informa sobre la fecha de lanzamiento.

\section{C.2.2 Especificación de la fecha de la última versión}

Todas las aplicaciones se van modificando y actualizando periódicamente dando lugar a diferentes versiones, (estas pueden variar según el sistema operativo y el dispositivo) en las que se van incorporando nuevas opciones, funcionalidades y mejoras que, en algunos casos, requerirán diferentes versiones también del sistema operativo.

\section{C.2.3 Especificación del historial de versiones}

Los cambios incorporados a las diferentes versiones, reflejan la preocupación del desarrollador por mejorar sus productos y seguir las recomendaciones hechas por los usuarios. 
App Store permite la consulta del historial de versiones, sin embargo ni Google Play ni Amazon nos ofrecen este dato, solo el de la última actualización.

\section{C.2.4 Incorporación frecuente de nuevos títulos}

Los títulos de una buena app deben actualizarse periódicamente para mantener la atención de los lectores y, aunque no existe una recomendación fija, en principio será mejor aquella que incorpore los títulos con una periodicidad menor. Por ejemplo, Play Tales incluye nuevos títulos cada semana, mientras que en otras como Joy Tales el último título incorporado es de hace dos años.

Esta información puede obtenerse en la web de los desarrolladores, en algunos casos como App Store en la propia descripción del producto y en otros no aparece por lo que será necesario consultar periódicamente la aplicación para ver si hay nuevos títulos.

\section{C.3 Contenidos}

De forma genérica valoraremos los contenidos incluidos en la aplicación, no solo la cantidad, sino también la variedad en temas, géneros y edades, siempre teniendo en cuenta que estamos evaluando la aplicación, no el contenido o la calidad literaria y artística de cada uno de los títulos, aspecto que debe ser abordado de manera individualizada aplicando criterios específicos para la literatura infantil y juvenil como los paratextos (tipografía, diseño de la página, cubierta...) la adecuación de la ilustración y su relación con la historia texto, el tema, los aspectos textuales (vocabulario, lenguaje, narración, estilo...) o la edad, ya desarrollados por especialistas en la materia como las autoras mencionadas en la introducción y que será objeto de un posterior trabajo centrado en la selección de libros app.

Determinar el número de títulos que componen una aplicación permitirá establecer sus posibilidades de compra, de uso y también de permanencia en el tiempo. Si un producto cuenta con un número importante de títulos y además se van incorporando otros nuevos, el usuario va a seguir utilizándola, mientras que aquellas que solo cuentan con 6 ó 7 libros, e incluso menos, una vez leídos todos, ya no será interesante para el lector y dejará de utilizarla hasta que se incorporen nuevos títulos.

\section{C.3.1 Variedad en temas, géneros y edades}

Hay muchas aplicaciones que están especializadas en un tema, género ó edad, por lo que en estos casos no podremos aplicar este indicador, pero en aquellas más generalistas, la variedad en la oferta en cuanto a estos factores, puede ser un elemento de valoración positivo.

\section{C.4 Accesibilidad}

A través de este parámetro comprobaremos las herramientas de la app para acceder de forma adecuada a los contenidos.

\section{C.4.1 Campos de búsqueda en la tienda}

Si hay algo que caracteriza a las apps de lectura es que son utilizadas tanto por niños como por adultos, por lo que la tienda debe incorporar la posibilidad de recuperar los títulos por diferentes filtros o campos que tengan en cuenta estos dos grupos de lectores.

En el caso de las aplicaciones para los más pequeños se recomienda que el sistema de búsqueda sea simple, la imagen de cubierta, y que se vaya haciendo más complejo a medida que aumenta la edad, de modo que a partir de los 8 años sea posible buscar, como mínimo, por autor, tema y edad.

\section{C.4.2 Información sobre los títulos}

Cuanto mayor sea la información que sobre cada uno de los títulos ofrece la app, mayor será su accesibilidad. Con una buena descripción se evitan confusiones acerca de lo que se esta comprando. Esta información debe ser adecuada en cantidad y pertinencia; autor/ilustrador, precio y resumen deberían ser obligatorios, aunque no siempre aparecen.

\section{C.4.3 Lectura sin conexión a internet}

Teniendo en cuenta que estamos trabajando con menores y que podemos haber establecido algún sistema de control parental, las apps deben permitir la lectura de los títulos sin conexión a internet.

En este caso no se tienen en cuenta los criterios relacionados con la accesibilidad para niños con dificultades visuales, auditivas, o cognitivas. No porque no sea importante, sino porque es un tema que requiere un tratamiento independiente y un trabajo conjunto de especialistas en cada necesidad especial para el desarrollo de aplicaciones accesibles. 


\section{C.5 Organización de los contenidos}

Otro de los parámetros que podemos analizar es la forma en la que se organizan y consecuentemente se visualizan los contenidos de la aplicación.

\section{C.5.1 Tipos de visualización}

En general el modo de visualización es una estantería personal que puede aparecer bajo el nombre de "mis cuentos", "mi biblioteca" o "mis libros", a modo de expositor de novedades de una biblioteca, donde se localizan las obras a través de las cubiertas o buscando un título concreto; en pocos casos se ofrece la visualización en forma de listado.

Para los más pequeños se recomienda la presentación de las cubiertas, ya que ésta es la primera información que tiene el lector sobre un libro, le ayuda a reconocer personajes o identificar contenidos y es, en el caso de los más pequeños, uno de los paratextos esenciales a la hora de elegir un libro.

\section{C.5.2 Posibilidad de cambiar el modo de visualización}

Valoraremos igualmente la posibilidad de cambiar la visualización del modo estantería al modo listado; si bien es una opción que no suele ser habitual, salvo en las aplicaciones generales con contenidos infantiles.

\section{C.5.3 Organización de carpetas}

A partir de 8 años se recomienda que las apps permitan también la opción de organizar los títulos en carpetas personalizadas por el lector, algo que le dará la posibilidad de categorizar los libros por temas y le facilitará posteriormente la recuperación. Esta opción solo suele estar presente en las aplicaciones generales con contenido infantil.

\section{C.5.4 Eliminación de títulos}

La posibilidad de eliminar libros para disponer de más memoria o simplemente para borrar aquellos que no respondan a nuestros intereses es imprescindible en cualquier aplicación. Esta eliminación debe ser realizada preferiblemente desde la estantería personal con un mensaje de confirmación, si bien existen algunas aplicaciones en las que esta eliminación se realiza desde el menú de configuración.

\section{C.5.5 Restauración de títulos}

Siempre es posible equivocarse al eliminar un título. Si la descarga ha sido gratuita o es de una app de suscripción podremos volverlo a descargar, pero si lo hemos comprado, es imprescindible que la app permita restaurar compras y comprobar también la facilidad o dificultad de esta operación.

\section{C.6 Personalización de los contenidos}

Las aplicaciones integran una serie de funcionalidades imposibles en la literatura impresa que permiten adaptar los contenidos a las preferencias de los lectores. Este parámetro solo se aplicará en aquellos casos en los que todos los títulos de la aplicación tengan las mismas funcionalidades y características. Dentro de este parámetro hay que considerar:

C.6.1 Posibilidad de personalizar los aspectos formales del texto

Valoraremos en este caso la posibilidad de cambiar el tipo y tamaño de la letra (a partir de 8 años); el ajuste del brillo y del fondo de pantalla y la lectura a una o doble página (solo en los libros de imágenes). Además hay que tener en cuenta si se puede cambiar el idioma del texto e incluso elegir entre las distintas variantes de un mismo idioma (inglés americano y británico).

\section{C.6.2 Posibilidad de personalizar los aspectos formales de audio}

En este caso tendremos en cuenta la opción de la locución de la historia a través de un narrador. Cuando esta opción se combina con distintos idiomas, se favorece el aprendizaje de las diferentes lenguas. Además es conveniente que las aplicaciones dirigidas a menores de 6 años permitan la grabación personalizada de la historia. Finalmente una buena aplicación debe permitir que el lector active/desactive tanto la banda sonora como los efectos de audio según sus preferencias.

\section{C.6.3 Posibilidad de personalizar los aspectos formales del vídeo y animación}

Es aconsejable que, en caso de existir, el movimiento de objetos y personajes no se active de forma automática sino que sea el lector el que decida. 


\section{C.6.4 Búsqueda en los contenidos}

Este indicador permitirá determinar la posibilidad de buscar palabras en un diccionario precargado en la app, a través de internet o en la Wikipedia, e incluso buscar la traducción en otros idiomas. Es una opción recomendable a partir de los 8 años, pero que dependerá del sistema de control parental y la posibilidad de acceder a determinadas páginas especificadas por el adulto.

\section{C.6.5 Anotaciones}

Realizar anotaciones durante el proceso de lectura es una actividad más propia del entorno científico y académico $y$, aunque no es muy habitual que los niños las realicen, a medida que vamos avanzando en las etapas lectoras se puede convertir en una opción útil. En este caso las acciones más interesantes son: subrayar y destacar; incluir y borrar notas; incluir y borrar marcadores y generar índice de notas y marcadores.

\section{C.7 Interacción con los contenidos}

Son cada vez más los niños y jóvenes que demandan poder compartir sus lecturas, valorarlas, hacer comentarios y ver que opinan sus semejantes. La recomendación de un amigo es para ellos uno de los motivos fundamentales por los que se deciden a comprar o descargar una aplicación sea del tipo que sea. Por eso, debemos comprobar si éstas permiten realizar este tipo de acciones a través de dos indicadores.

\section{C.7.1 Posibilidad de compartir comentarios o valoraciones}

Determinaremos en este caso si es posible compartir y valorar la aplicación y a través de qué medio, por ejemplo correo electrónico o redes sociales, siempre teniendo en cuenta que esta opción suele estar limitada a los adultos y que en las aplicaciones para los más pequeños ni siquiera aparece.

Dado que los niños son poco proclives a escribir textos extensos, son recomendables aquellas que incluyen un mensaje tipo ya redactado en el que solo hay que añadir la dirección del destinatario.

\section{C.7.2 Posibilidad de puntuar}

Es aconsejable que el lector tenga la posibilidad de puntuar la aplicación a través de la asignación de puntos o estrellas. Se puede puntuar desde la tienda o directamente desde la aplicación, e incluso cada uno de los títulos.

\section{C.8 Calidad del audio}

Este parámetro sólo se valorará en aquellas aplicaciones en las que todos sus títulos tengan las mismas funcionalidades de audio. Los indicadores son:

\section{C.8.1 Tipo de audio}

Especificaremos si incluye narración, banda sonora (música incorporada a las diferentes historias) y efectos de sonido (aquellos que se activan al pulsar sobre un objeto o personaje).

\section{C.8.2 Calidad de la narración}

Comprobaremos si el narrador modula la voz de forma correcta, si es una voz neutra o tiene alguna entonación regional y si hay dramatización.

\section{C.9 Interactividad}

Muchos expertos señalan que ésta es una de las características fundamentales de la edición digital ya que permite al lector actuar y participar como un personaje más de la historia y tomar decisiones en el proceso de lectura. Por ello es conveniente comprobar si la aplicación evaluada incluye animación e interactividad, si está equilibrada, si es excesiva... como en otros casos solo se tendrá en cuenta cuando todos los títulos de la aplicación tienen esta funcionalidad.

\section{C.9.1 Existencia de interactividad}

Indicaremos simplemente la existencia o no de interactividad y animación en los títulos que componen la aplicación, sin olvidarnos que un buen diseño de la interactividad (adecuado y proporcionado) es un valor añadido a la aplicación porque permite al lector no perderse en la lectura, aunque puede haber aplicaciones de calidad cuyos títulos no incluyan este recurso. 


\subsection{Desarrollo de la herramienta de trabajo}

Antes de evaluar es necesario tener en cuenta distintas consideraciones:

- La valoración de una aplicación ha de hacerse con la suma de todos los parámetros. No se puede rechazar un producto por el hecho de que no cumpla alguno de los considerados recomendables.

- Algunos de los parámetros propuestos pueden ser utilizados para evaluar cualquier aplicación para niños (accesibilidad, usabilidad, etc.) y otros son propios y específicos de las aplicaciones de lectura e incluso de los libros app.

- Todos los parámetros e indicadores deben valorarse atendiendo a la edad de los posibles lectores. La literatura infantil tiene en cuenta la edad de sus destinatarios, sus capacidades lectoras y sus características psicológicas $\mathrm{y}$, aunque sea un criterio sobre el que hay opiniones confrontadas y debamos utilizar la edad como una simple orientación, puede servirnos de referencia a la hora de seleccionar. Determinar la edad de los potenciales lectores nos permitirá comprobar si las funcionalidades y características de cada aplicación son las adecuadas ya que algunos aspectos que pueden considerarse como necesarios en ciertas etapas pueden no tener importancia en otras. Por ejemplo, es recomendable que una aplicación para 13 ó 14 años permita anotaciones y subrayados, pero no es necesario en las destinadas a los de 5 ó 6 años; por el contrario la posibilidad de personalizar la narración, tan útil para los más pequeños, no se considera esencial en etapas superiores.

- Existen indicadores que no se pueden medir de forma efectiva. Por ejemplo, la visibilidad puede cuantificarse en función de la presencia en fuentes y recursos especializados, algo que llevaría gran cantidad de tiempo, por lo que será necesario recurrir a blogs y recomendadores especializados si queremos analizar determinados parámetros.

En la actualidad las únicas herramientas con las que contamos para seleccionar aplicaciones son los blogs y recomendadores especializados ${ }^{12}$ que realizan una labor de análisis y evaluación, para la cual pueden ser especialmente útiles la sistematización de parámetros e indicadores que faciliten su tarea y permitan la comparación.

A continuación se muestra una plantilla que servirá de guía para la evaluación de las aplicaciones. Esta dividida en tres apartados; en el primero se recoge la información general de la aplicación y en los dos siguientes cada una de las dos dimensiones, seleccionando los parámetros, indicadores y en caso necesario los subindicadores asociados. Cada uno de ellos se valorará positivamente (1) o no se valorará (0). En la columna de observaciones se indica cuando es imprescindible o recomendable la presencia de este indicador así como algunas peculiaridades en función de las edades a las que esten destinadas.

La puntuación global se puede obtener dividiendo el total de puntos obtenidos entre el número de indicadores aplicados. Si estamos ante un desarrollo en el que no hay locución, no se puede valorar la calidad, por lo que el número de indicadores en cada caso será variable.

Se incluye también un apartado titulado valoración final en la que el evaluador redactará una reseña sobre el producto en base al análisis global de todos los parámetros e indicadores. Esta reseña es la que se ofrecerá a los lectores por parte de los bibliotecarios, maestros, blogueros...

La suma de puntos obtenidos permitirá establecer tres niveles de aplicaciones:

a) Altamente recomendables: presencia del 80\% de los indicadores evaluables en cada caso, considerados imprescindibles y recomendables.

b) Recomendables: entre el 50 y el 79\% de los indicadores evaluables en cada caso, considerados imprescindibles y recomendables.

c) Prescindibles: menos del $49 \%$ de los indicadores evaluables en cada caso, considerados imprescindibles y recomendables.

\begin{tabular}{|c|c|c|c|c|c|c|c|}
\hline \multicolumn{8}{|c|}{ DATOS GENERALES DE LA APLICACIÓN } \\
\hline \multicolumn{8}{|c|}{$\begin{array}{l}\text { DATOS GENERALES DE LA APLICACION } \\
\text { APLICACIÓN }\end{array}$} \\
\hline \multicolumn{8}{|c|}{$\begin{array}{l}\text { APLICACION } \\
\text { DESARROLLADOR }\end{array}$} \\
\hline \multicolumn{4}{|c|}{ PRECIO } & \multicolumn{4}{|c|}{ FECHA DE LA VERSIÓN ANALIZADA } \\
\hline \multicolumn{8}{|c|}{ 至 } \\
\hline \multicolumn{8}{|c|}{ REQUIRIMIENTOS } \\
\hline IDIOMA/S & Español & Inglés & Francés & Catalán & Gallego & Euskera & Otros \\
\hline \multicolumn{8}{|c|}{ TIPOS DE CONTENIDOS (cuentos populares, de animales, informativos, etc.) } \\
\hline EDAD & & & & & & & \\
\hline
\end{tabular}




\begin{tabular}{|c|c|c|c|c|c|}
\hline \multicolumn{6}{|l|}{ DIMENSIÓN FORMA } \\
\hline PARÁMETRO & INDICADORES & & NO $=0$ & SI $=1$ & Observaciones \\
\hline \multirow[t]{7}{*}{ DISPONIBILIDAD } & \multirow{3}{*}{$\begin{array}{l}\text { Disponibilidad para } \\
\text { diferentes sistemas } \\
\text { operativos }\end{array}$} & Android & & & \\
\hline & & IOS & & & \\
\hline & & Otros & & & \\
\hline & \multicolumn{2}{|c|}{ Disponibilidad en diferentes sitios } & & & Recomendable \\
\hline & \multicolumn{2}{|c|}{$\begin{array}{l}\begin{array}{l}\text { Disponibilidad de la app en varios } \\
\text { idiomas }\end{array} \\
\end{array}$} & & & \\
\hline & \multicolumn{2}{|c|}{ Título identificable } & & & Imprescindible \\
\hline & \multicolumn{2}{|l|}{ Icono identificable } & & & Imprescindible \\
\hline \multirow[t]{8}{*}{ ADQUISICIÓN } & \multirow[t]{2}{*}{ Precio } & Gratuita & & & \\
\hline & & $\begin{array}{l}\text { Precio unitario de } \\
\text { los títulos }\end{array}$ & & & \\
\hline & \multirow{6}{*}{$\begin{array}{llr}\text { Modalidad } & \text { de } \\
\text { adquisición } & \text { de } & \text { los } \\
\text { contenidos } & & \end{array}$} & $\begin{array}{l}\text { Compras } \\
\text { integradas }\end{array}$ & & & \\
\hline & & Suscripción & & & \\
\hline & & $\begin{array}{ll}\begin{array}{l}\text { Gratuita } \\
\text { publicidad }\end{array} & \text { con } \\
\end{array}$ & & & No recomendable \\
\hline & & $\begin{array}{ll}\text { Gratuita } & \text { sin } \\
\text { publicidad }\end{array}$ & & & \\
\hline & & $\begin{array}{l}\text { Versión lite de } \\
\text { algunos títulos }\end{array}$ & & & \\
\hline & & $\begin{array}{l}\text { Ofertas } \\
\text { (descuentos } \quad \text { o } \\
\text { packs) }\end{array}$ & & & \\
\hline \multirow[t]{3}{*}{ SEGURIDAD } & \multicolumn{2}{|c|}{$\begin{array}{l}\text { Bloqueo de compras en la propia } \\
\text { aplicación }\end{array}$} & & & Imprescindible \\
\hline & \multicolumn{2}{|c|}{ Control parental en la propia aplicación } & & & $\begin{array}{l}\text { Imprescindible salvo } \\
\text { que no incluya } \\
\text { compras integradas }\end{array}$ \\
\hline & \multicolumn{2}{|c|}{$\begin{array}{l}\text { Clave de acceso adaptada a la edad del } \\
\text { usuario }\end{array}$} & & & Imprescindible \\
\hline \multirow[t]{2}{*}{$\begin{array}{l}\text { COFINDENCIALIDAD } \\
\text { PRIVACIDAD }\end{array}$} & \multicolumn{2}{|c|}{$\begin{array}{l}\text { Explicación clara de la política de } \\
\text { privacidad }\end{array}$} & & & Imprescindible \\
\hline & \multicolumn{2}{|c|}{ Anonimato de los menores } & & & Imprescindible \\
\hline POPULARIDAD & \multicolumn{2}{|c|}{ Puntuación de los lectores } & & & $\begin{array}{lr}\text { Solo valorar } \\
\text { positivamente } \\
\text { partir de 3 puntos }\end{array}$ \\
\hline \multirow[t]{2}{*}{ RECONOCIMIENTO } & \multicolumn{2}{|l|}{ Premios recibidos } & & & Indicar premios \\
\hline & \multicolumn{2}{|l|}{ Sellos de calidad } & & & Indicar sellos \\
\hline \multirow[t]{6}{*}{ USABILIDAD } & \multirow[t]{2}{*}{ Velocidad } & Apertura rápida & & & $\begin{array}{l}\text { Recomendación } 10 \text { a } \\
25 \text { segundos }\end{array}$ \\
\hline & & $\begin{array}{lr}\text { Mensajes } & \text { del } \\
\text { proceso } & \text { de } \\
\text { descarga } & \text { durante } \\
\text { los } & \text { tiempos } \\
\text { muertos } & \\
\end{array}$ & & & \\
\hline & \multirow[t]{4}{*}{ Navegación } & $\begin{array}{l}\text { Navegación es } \\
\text { sencilla e intuitiva }\end{array}$ & & & Recomendable \\
\hline & & $\begin{array}{l}\text { Funcionamiento } \\
\text { correcto }\end{array}$ & & & Indicar los fallos \\
\hline & & $\begin{array}{l}\text { Iconos de los } \\
\text { botones de acción } \\
\text { identificables }\end{array}$ & & & Imprescindible \\
\hline & & Existencia de & & & \\
\hline
\end{tabular}




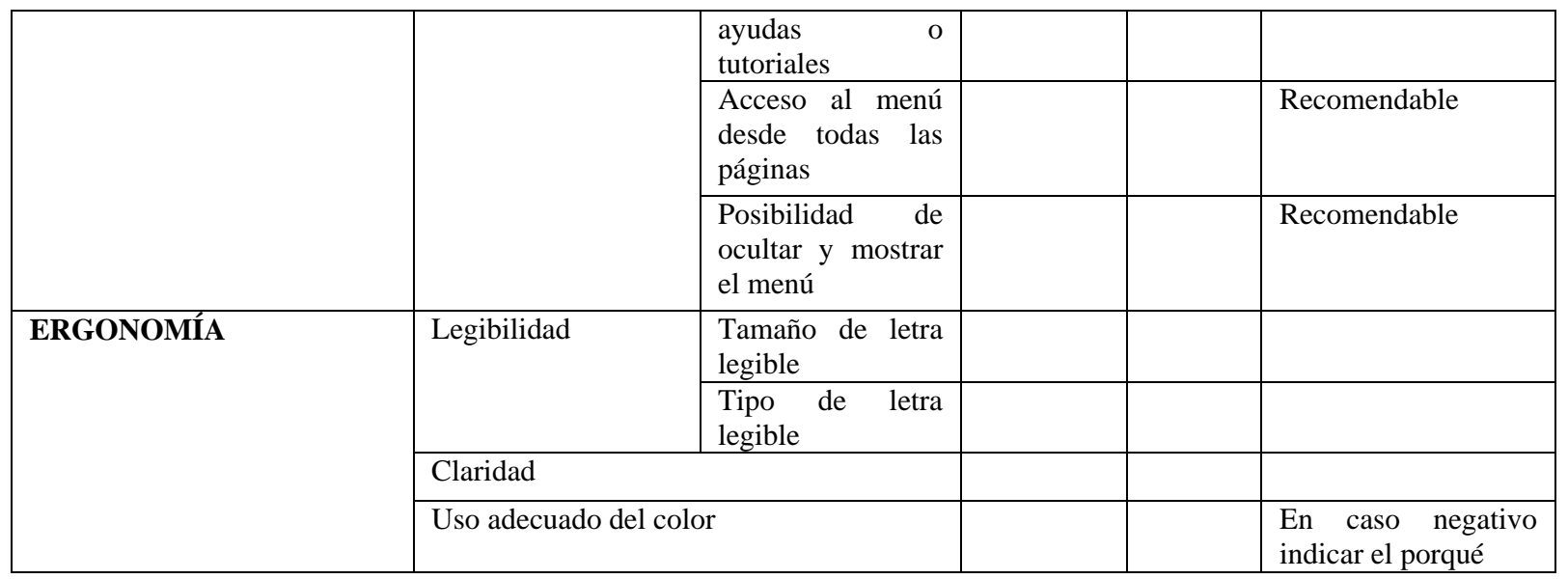

\begin{tabular}{|c|c|c|c|c|c|c|}
\hline \multicolumn{7}{|c|}{ DIMENSIÓN CONTENIDO } \\
\hline PARÁMETRO & INDICADORE & & & NO $=0$ & SI $=1$ & Observaciones \\
\hline AUTORÍA & \multicolumn{3}{|c|}{ Mención del editor/desarrollador } & & & Imprescindible \\
\hline \multirow[t]{4}{*}{ ACTUALIZACIÓN } & \multicolumn{3}{|c|}{ Especificación de la fecha de creación } & & & Imprescindible \\
\hline & \multicolumn{3}{|c|}{$\begin{array}{l}\text { Especificación de la fecha de la última } \\
\text { versión }\end{array}$} & & & \\
\hline & \multicolumn{3}{|c|}{ Especificación del historial de versiones } & & & \\
\hline & \multicolumn{3}{|c|}{$\begin{array}{llll}\text { Incorporación } & \text { frecuente } & \text { de } & \text { nuevos } \\
\text { títulos } & & \\
\end{array}$} & & & Recomendable \\
\hline CONTENIDOS & \multicolumn{3}{|c|}{ Variedad en temas, géneros y edades } & & & $\begin{array}{l}\text { Solo valorable en las } \\
\text { generales }\end{array}$ \\
\hline \multirow[t]{7}{*}{ ACCESIBILIDAD } & \multirow{3}{*}{\multicolumn{2}{|c|}{$\begin{array}{llll}\text { Campos } & & \text { de } \\
\text { búsqueda } & \text { en } & \text { la } \\
\text { tienda } & & \\
\end{array}$}} & $\begin{array}{l}\text { Búsqueda por } \\
\text { autor }\end{array}$ & & & $\begin{array}{l}\text { Recomendable a partir de } \\
8 \text { años }\end{array}$ \\
\hline & & & $\begin{array}{l}\text { Búsqueda por } \\
\text { tema }\end{array}$ & & & $\begin{array}{l}\text { Recomendable a partir de } \\
8 \text { años }\end{array}$ \\
\hline & & & $\begin{array}{l}\text { Búsqueda } \\
\text { edad }\end{array}$ & & & $\begin{array}{l}\text { Recomendable a partir de } \\
8 \text { años }\end{array}$ \\
\hline & \multirow{3}{*}{\multicolumn{2}{|c|}{$\begin{array}{l}\text { Información sobre } \\
\text { los títulos }\end{array}$}} & Autor/ilustrador & & & Imprescindible \\
\hline & & & Precio & & & Imprescindible \\
\hline & & & Resumen & & & Imprescindible \\
\hline & \multicolumn{3}{|c|}{ Lectura sin conexión a internet } & & & Recomendable \\
\hline \multirow{7}{*}{$\begin{array}{l}\text { ORGANIZACIÓN DE } \\
\text { LOS CONTENIDOS }\end{array}$} & \multirow{2}{*}{\multicolumn{2}{|c|}{$\begin{array}{ll}\text { Tipos } & \text { de } \\
\text { visualización }\end{array}$}} & Estantería & & & Imprescindible \\
\hline & & & Listado & & & \\
\hline & \multicolumn{3}{|c|}{$\begin{array}{l}\text { Posibilidad de cambiar el modo de } \\
\text { visualización }\end{array}$} & & & \\
\hline & \multicolumn{3}{|c|}{ Organización de carpetas } & & & $\begin{array}{l}\text { Solo valorable a partir de } \\
8 \text { años }\end{array}$ \\
\hline & \multirow{2}{*}{\multicolumn{2}{|c|}{$\begin{array}{l}\text { Eliminación de } \\
\text { títulos }\end{array}$}} & $\begin{array}{l}\text { Posibilidad de } \\
\text { eliminar títulos }\end{array}$ & & & Imprescindible \\
\hline & & & $\begin{array}{l}\text { Eliminación } \\
\text { directa desde la } \\
\text { estantería }\end{array}$ & & & Recomendable \\
\hline & \multicolumn{3}{|c|}{ Restauración de títulos } & & & Imprescindible \\
\hline \multirow{3}{*}{$\begin{array}{l}\text { PERSONALIZACIÓN } \\
\text { DE LOS } \\
\text { CONTENIDOS }\end{array}$} & \multirow{3}{*}{$\begin{array}{l}\text { Posibilidad de } \\
\text { personalizar } \\
\text { los aspectos } \\
\text { formales de } \\
\text { texto }\end{array}$} & \multicolumn{2}{|c|}{ Tipo y tamaño de letra } & & & $\begin{array}{l}\text { Solo valorable a partir de } \\
8 \text { años }\end{array}$ \\
\hline & & \multicolumn{2}{|c|}{$\begin{array}{l}\text { Ajuste de brillo } \mathrm{y} \\
\text { fondo de pantalla }\end{array}$} & & & Imprescindible \\
\hline & & \multicolumn{2}{|c|}{ Lectura a doble página } & & & $\begin{array}{l}\text { Solo valorable en los } \\
\text { libros de imágenes }\end{array}$ \\
\hline
\end{tabular}




\begin{tabular}{|c|c|c|c|}
\hline & & $\begin{array}{l}\text { Cambio del idioma del } \\
\text { texto }\end{array}$ & Recomendable \\
\hline & & Narración oral & \\
\hline & $\begin{array}{l}\text { personallzar } \\
\text { los aspectos }\end{array}$ & $\begin{array}{l}\text { Cambio del idioma de } \\
\text { la narración }\end{array}$ & Recomendable \\
\hline & audio & $\begin{array}{l}\text { Grabación } \\
\text { personalizada }\end{array}$ & $\begin{array}{l}\text { Recomendable } \\
\text { menores de } 6 \text { años }\end{array}$ \\
\hline & & $\begin{array}{l}\text { Activación/desactivaci } \\
\text { ón de efectos de audio }\end{array}$ & Imprescindible \\
\hline & $\begin{array}{l}\text { Posibilidad de pe } \\
\text { formales del víde }\end{array}$ & $\begin{array}{l}\text { ersonalizar los aspectos } \\
\text { o y animación }\end{array}$ & Recomendable \\
\hline & $\begin{array}{l}\text { Búsqueda en } \\
\text { los contenidos }\end{array}$ & $\begin{array}{ll}\text { Búsqueda } & \text { en } \\
\text { diccionario } & \\
\end{array}$ & $\begin{array}{l}\text { Recomendable a partir de } \\
8 \text { años }\end{array}$ \\
\hline & & Búsqueda en internet & $\begin{array}{l}\text { Recomendable a partir de } \\
8 \text { años }\end{array}$ \\
\hline & & $\begin{array}{l}\text { Búsqueda en } \\
\text { Wikipedia }\end{array}$ & $\begin{array}{l}\text { Recomendable a partir de } \\
8 \text { años }\end{array}$ \\
\hline & Anotaciones & Subrayar y destacar & $\begin{array}{l}\text { Recomendable a partir de } \\
8 \text { años }\end{array}$ \\
\hline & & Incluir/borrar notas & $\begin{array}{l}\text { Recomendable a partir de } \\
8 \text { años }\end{array}$ \\
\hline & & $\begin{array}{l}\text { Incluir/borrar } \\
\text { marcadores }\end{array}$ & $\begin{array}{l}\text { Recomendable a partir de } \\
8 \text { años }\end{array}$ \\
\hline & & $\begin{array}{l}\text { Índice de notas } \mathrm{y} \\
\text { marcadores }\end{array}$ & $\begin{array}{l}\text { Recomendable a partir de } \\
8 \text { años }\end{array}$ \\
\hline $\begin{array}{l}\text { INTERACCIÓN CON } \\
\text { LOS CONTENIDOS }\end{array}$ & $\begin{array}{l}\text { Posibilidad de cc } \\
\text { valoraciones }\end{array}$ & ompartir comentarios y & \\
\hline & Posibilidad de pu & intuar & \\
\hline CALIDAD DEL AUDIO & Tipo de audio & Narración & \\
\hline & & Banda sonora & \\
\hline & & Efectos de sonido & \\
\hline & $\begin{array}{l}\text { Calidad de la } \\
\text { narración }\end{array}$ & $\begin{array}{l}\text { Entonación y } \\
\text { modulación correcta }\end{array}$ & Imprescindible \\
\hline & & Dramatización & \\
\hline INTERACTIVIDAD & $\begin{array}{l}\text { Existencia de } \\
\text { interactividad }\end{array}$ & & \\
\hline
\end{tabular}

Tabla III. Plantilla para la evaluación.

\section{CONCLUSIONES}

Teniendo en cuenta el elevado número de aplicaciones, su selección y posterior recomendación se hace cada vez más necesaria para ahorrar tiempo y por qué no decirlo dinero a los lectores, pero fundamentalmente porque es una herramienta no solo para el aprendizaje sino también para la promoción y difusión de la lectura, que permitirá orientar a los mediadores, objetivar las decisiones y compartir con otros las valoraciones. No se trata solo de poner la tecnología al alcance de los niños sino poner en sus manos los mejores productos.

Los trabajos de evaluación sobre aplicaciones para el público infantil son escasos y aunque existen estudios ya publicados la mayoría no son específicos de las aplicaciones para la lectura infantil.

Una vez realizado este trabajo hemos podido constatar la necesidad de definir parámetros e indicadores más o menos objetivables que permitan a los profesionales poner en marcha servicios de recomendación de aplicaciones al igual que se viene haciendo tradicionalmente con la literatura impresa.

Hemos de mencionar igualmente el elevado número de parámetros que es necesario considerar a la hora de evaluar una app de lectura infantil. Su aplicación práctica supondrá un coste temporal importante pero amortizable con la 
puesta en marcha de nuevos servicios bibliotecarios centrados en la literatura infantil digital: la hora del cuento digital, talleres con los padres, presentaciones de aplicaciones....

Asimismo hay que tener en cuenta la imposibilidad para medir algunos parámetros e indicadores por la falta de información suministrada bien por las propias tiendas e incluso los propios desarrolladores. Un aspecto que impide que en muchas ocasiones la evaluación sea todo lo completa que sería deseable.

Siempre ha habido y habrá grandes obras de la literatura que no han encontrado su lector independientemente de su calidad y en el caso de las aplicaciones puede ocurrir exactamente lo mismo. Nuestra labor es conseguir que cada aplicación encuentre su usuario.

\section{NOTAS}

1 En este sentido pueden consultarse en el Instituto Nacional de Estadística los datos correspondientes al año 2014 <http://www.ine.es/jaxi/menu.do?type=pcaxis\&path=/t25/p450/base_2011/a2014/\&file=pcaxis> donde se indica el número de hogares con tabletas y el uso de las mismas por parte de los menores.

${ }^{2}$ Véase <http://www.statista.com/statistics/276623/number-of-apps-available-in-leading-app-stores/>.

${ }^{3}$ Véase <http://www.statista.com/statistics/270291/popular-categories-in-the-app-store/>.

4 <http://literatura.gretel.cat/es/recomanacions-digitals>.

5 <http://www.canallector.com/docs/281/Guia-de-Apps->.

${ }^{6}$ La descubribilidad es conocer la existencia de algo, en nuestro caso la capacidad de que los interesados en una aplicación la encuentren de una forma fácil y sencilla a través de muchos sitios.

${ }^{7}$ Todos los expertos afirman que el título de un producto es fundamental para la optimización y visibilidad en una tienda de aplicaciones. Es un indicador fundamental de la ASO (App Store Optimization). Cuanto mejor sea el posicionamiento ASO de una aplicación, más visible será para los clientes potenciales y más descargas se producirán.

${ }^{8}$ Los bundles son paquetes de aplicaciones formados por varios productos que se compran de forma conjunta.

9 No confundir con las compras integradas de personajes, objetos, stikers... propias de juegos o storytelling.

${ }^{10}$ Las redes de publicidad móvil no permiten filtrar por público infantil por lo que pueden estar viendo publicidad no regulada e incluso peligrosa.

${ }^{11}$ Según el último informe de la Agencia Nacional de Protección de Datos (2014), el 31\% de las aplicaciones ofrece "cierta información" sobre la utilización de los datos personales de sus clientes, el 24\% da información "inadecuada" y el 30\% restante no ofrece ningún dato más allá de los permisos que se solicitan al instalar la aplicación. En el 59\% de las apps analizadas, los usuarios encuentran dificultades para hallar antes de instalarlas la información relativa a la privacidad. El mismo informe establece igualmente que el 31\% de ellas solicita al usuario excesivos permisos en comparación con la función que desempeñan.

${ }^{12}$ Véase por ejemplo: Frikids, Cappaces, Appsmama, Digital Story Time, Mom with apps, Smartappforkids, La Sourise Grise, Appsmama.es, Apps Playground, Bestappsforkids, Generación Apps, Literatura Exploratorias, Probando Apps, El Blog de Elisa Yuste y Proyecto Guappis.

\section{BIBLIOGRAFÍA}

AGENCIA Española de Protección de Datos. Resultados del análisis coordinado sobre las condiciones de privacidad de las aplicaciones móviles. $2014 . \quad$ Disponible en: <http://www.agpd.es/portalwebAGPD/revista_prensa/revista_prensa/2014/notas_prensa/common/sep_14/140910_ NP_Resultados_analisis_GPEN.pdf> [Consulta: 11 de abril de 2015].

BIRCHER, K. What makes a good picture book app. The Horn Book Magazine, 2012, vol. 88, $\mathrm{n}^{\circ}$ 2, p. 72. Disponible en: <http:/www.hbook.com/2012/02using-books/what-makes-a-good-picture-book-app/\#_> [Consulta: 10 de mayo de 2015].

CAHILL, M. y MCGILL - FRANZEN, A. Selecting “app” ealing and “app” ropriate book apps for beginning readers. The Reading Teacher, 2013, vol. 67, $\mathrm{n}^{\circ}$ 1, p. 30-39.

CODINA, L. Evaluación de recursos digitales en línea: conceptos, indicadores y métodos. Revista española de documentación científica, 2000, vol. 23, $\mathrm{n}^{\circ} \quad 1$, p. $9-44 . \quad$ Disponible en: $<$ http://redc.revistas.csic.es/index.php/redc/article/viewFile/315/479> [Consulta: 25 de marzo de 2015].

CODINA, L. Evaluación de calidad en sitios web: Metodología de proyectos de análisis sectoriales y de realización de auditorías. Barcelona: UPF, 2006. Disponible en: <http://eprints.rclis.org/8854/1/procedimientos2006.pdf> [Consulta: 25 de marzo de 2015].

COLOMER, T. Introducción a la literatura infantil y juvenil actual. Madrid: Síntesis, 2010.

GARCÍA RODRIGUEZ, A. Aplicaciones de lectura infantil y competencias digitales: evaluar antes de enseñar. Vegajournal 2014, vol. 10, $\mathrm{n}^{\circ}$ 1, p. 21-39. Disponible en: <http://www.vegajournal.org/content/archivio/66-201404-anno-X-numero-1/317-aplicaciones-de-lectura-infantil-y-competencias-digitales-evaluar-antes-de-ensenar $>$ [Consulta: 27 de marzo de 2015]. 
GARCÍA RODRÍGUEZ, A.; GÓMEZ-DÍAZ, R. y CORDÓN-GARCÍA, J.A. De Alicia en el país de las maravillas a las maravillas de Alicia: o de cómo cambia la selección de libros en papel a libros electrónicos infantiles y juveniles. Revista Ibersid, 2013, vol. 16, p. 21-32. Disponible en: <http://www.ibersid.eu/ojs/index.php/ibersid/article/view/4092> [Consulta: 26 de marzo de 2015].

GÓMEZ-DÍAZ, R. La evaluación de las aplicaciones de lectura. Cordón, J.A.; Carbajo, F.; Gómez, R. y Alonso, J. (Coords). Libros electrónicos y contenidos digitales en la sociedad del conocimiento: mercado, servicios y derechos. Madrid: Pirámide, 2012, p. 294-295.

GÓMEZ-DÍAZ, R. y CORDÓN-GARCÍA, J.A. Aplicaciones de lectura. DINLE: Diccionario Digital de nuevas formas de lectura y escritura. Universidad de Salamanca. Red Internacional de Universidades Lectoras. 2015. Disponible en: <http://dinle.usal.es/searchword.php?valor=Aplicaciones de lectura> [Consulta: 11 de marzo de 2015].

GUILLEM, J.A. y CELAYA, J. Apps educativas: nuevas formas de acceso al conocimiento. Madrid, Dosdoce. 2014.

GUILLEN, J.F. y AYUSO, M.D. Evaluación de la calidad de las bases de datos en línea útiles para Enfermería: propuesta teórico-metodológica. Anales de Documentación, 2014, vol. 17, $\mathrm{n}^{0} \quad 2$. Disponible en: <http://revistas.um.es/analesdoc/article/view/200691> [Consulta: 25 de marzo de 2015].

HENNIG, N. Selecting and Evaluating the Best Mobile Apps for Library Services. Library Technology Reports, 2014, vol. 50, no 8. Disponible en: <http://journals.ala.org/ltr/issue/view/180> [Consulta: 13 de abril de 2015].

HO, H.; LU, M. y LIN, P. The diverse perceived qualities and perceived values on app e-book and app e-magazine. Pakistan Journal. Statistic, 2013, vol. 29, nº 6, p. 1029-1048.

LLUCH, G. et al. Cómo reconocer los buenos libros para niños y jóvenes. Orientaciones de una investigación de Fundalectura (Colombia). Bogotá: Fundalectura, 2009.

LLUCH, G. Cómo seleccionar libros para niños y jóvenes. Los comités de valoración en las bibliotecas escolares y públicas. Gijón: Trea, 2010.

MANGAS-VEGA, A. Approach to the evaluation of electronic scientific monographs. En: Proceedings of the Second International Conference on Technological Ecosystems for Enhancing Multiculturality. ACM, New York, 2014. p. 537-540.

RELLÓ, L. y BAEZA-YATES, R. Evaluation of DysWebxia: a reading app designed for people with dyslexia. En: Proceedings of the 11th Web for All Conference. ACM, New York, 2014.

RODRÍGUEZ YUNTA, L. Evaluación e indicadores de calidad en bases de datos. Revista española de documentación científica, 1998, vol. 21, n 1, p. 9-23.

RODRÍGUEZ YUNTA, L. y GIMÉNEZ TOLEDO, E. Más allá de la usabilidad: características mínimas exigibles para las interfaces de bases de datos web. BiD: Textos universitaris de biblioteconomia i documentació, 2004, $\mathrm{n}^{\circ}$ 13. Disponible en: <http://bid.ub.edu/13rodri2.htm> [Consulta: 30 de marzo de 2015].

RODRÍGUEZ BRAVO, B. y ALVITE DÍEZ, M. Propuesta metodológica de evaluación de interfaces de OPACs. INNOPAC versus UNICORN. Revista española de documentación científica, 2004, vol. 27, $\mathrm{n}^{\circ} 1$. Disponible en: $<$ http://redc.revistas.csic.es/index.php/redc/article/viewArticle/147> [Consulta: 1 de abril de 2015].

RODRÍGUEZ MARTÍNEZ, R.; CODINA, L. y PEDRAZA JIMÉNEZ, R. Indicadores para la evaluación de la calidad en cibermedios: análisis de la interacción y de la adopción de la Web 2.0. Revista española de documentación científica, 2012, vol. 35, $\mathrm{n}^{\circ}$ 1, p. 61-93.

SÁNCHEZ, F.M. et al. (2002). Herramienta de evaluación de multimedia didáctico. Disponible en: $<$ http://www.sav.us.es/pixelbit/pixelbit/articulos/n18/n18art/art187.htm> [Consulta: 5 de abril de 2015].

SANZ PILAR, N. y GARCÍA RODRÍGUEZ, A. Los desarrolladores de libros apps infantiles y juveniles: radiografía, perspectivas y modelo de negocio, Anales de Documentación, 2014, vol. 17, $\mathrm{n}^{\circ}$ 2. Disponible en: <http://dx.doi.org/10.6018/analesdoc.17.2.192131> [Consulta: 19 de abril de 2015].

ZAID, G. Los demasiados libros. Barcelona: Debolsillo, 2010. 